\title{
FIB-4 score and hepatocellular carcinoma risk after hepatitis C virus cure: time to revise surveillance?
}

\author{
Antonio Saviano ${ }^{1,2,3}$, Simona Tripon ${ }^{1,2}$, Thomas F. Baumert ${ }^{1,2,3,4}$ \\ ${ }^{1}$ Institut de Recherche sur les Maladies Virales et Hépatiques (IVH), Inserm U1110, Strasbourg, France; ${ }^{2}$ Institut Hospitalo-Universitaire, Pôle \\ Hépato-digestif, Hôpitaux Universitaires de Strasbourg, Strasbourg, France; ${ }^{3}$ University of Strasbourg, Strasbourg, France; Institut Universitaire de \\ France (IUF), Paris, France \\ Correspondence to: Prof. Thomas F. Baumert, MD. Inserm U1110, Université de Strasbourg, 3 Rue Koeberlé, F-67000 Strasbourg, France. \\ Email: thomas.baumert@unistra.fr. \\ Comment on: Ioannou GN, Beste LA, Green PK, et al. Increased Risk for Hepatocellular Carcinoma Persists Up to 10 Years After HCV Eradication \\ in Patients With Baseline Cirrhosis or High FIB-4 Scores. Gastroenterology 2019;157:1264-78.e4.
}

Submitted Dec 24, 2019. Accepted for publication Jan 08, 2020.

doi: 10.21037/hbsn.2020.01.05

View this article at: http://dx.doi.org/10.21037/hbsn.2020.01.05

Hepatitis $\mathrm{C}$ virus (HCV) is a main cause and important risk factor for hepatocellular carcinoma (HCC) in Western countries (1). Direct antiviral agents (DAAs) for HCV are highly effective and well-tolerated antiviral drugs which allow $\mathrm{HCV}$ cure even in patients with advanced liver disease. However, whilst HCV cure improves liver function and decreases overall HCC risk, a significant HCC risk persists in particular in patients with advanced fibrosis including cirrhosis who have the highest risk of developing HCC (2-4).

Current international guidelines suggest that HCC surveillance programs, consisting in biannual ultrasound surveillance, should be offered to all HCV patients affected by cirrhosis $(5,6)$. Nevertheless, it is not clear whether noncirrhotic patients, e.g., with fibrosis graded Metavir F3, should be included nor whether these programs should be offered indefinitely to cirrhotic patients after $\mathrm{HCV}$ cure. Even though resolution or reduction of fibrosis has been observed after viral cure, it is unknown whether this translates in a reduction of HCC risk over-time. Moreover, $\mathrm{HCC}$ risk can change over time because of $\mathrm{HCV}$ independent risk factors such as age, alcohol intake, obesity and diabetes.

FIB-4 is a simple and easy-to-use score composed by patient's age, ALT, AST and platelet level and it has been developed and validated as non-invasive marker of liver fibrosis. An elevated FIB-4 score $(\geq 3.25)$ has a high specificity $(>96 \%)$ for advanced liver fibrosis (Ishak stage
4-6 or Metavir F3-F4) while a low FIB-4 score $(\leq 1.45)$ is associated with low fibrosis stages (Ishak stage 1-3 or Metavir F0-F2) (7). The FIB-4 score has been already shown to correlate with HCC risk in non-cirrhotic HCV patients treated with DAA and in patients with chronic $\mathrm{HBV}$, non-alcoholic fatty liver disease (NASH) and alcoholic cirrhosis (8-11).

In a recent study published in the journal Gastroenterology, Ioannou et al. analyzed patients' data from the Veterans Health Administration (VHA), one of the first US center to adopt an unrestricted access to DAAs with more than $50,000 \mathrm{HCV}$ patients treated in 15 years (2000-2015) who obtained a sustained viral response (SVR) $(12,13)$. Using individual laboratory data and ICD-9 and ICD-10 diagnosis records, Ioannou and colleagues calculated the HCC risk after SVR in cirrhotic and non-cirrhotic patients stratifying them according to anti-viral regimen (IFN or DAA) and pre-treatment and post-SVR FIB-4 score (13). Post-SVR FIB-4 changes were analyzed both as categorical variable at 1 -year post-SVR and as a time-dependent factor (13). Since some studies had reported an increased HCC risk associated with DAA treatment $(14,15)$, it is important to note that this study included only patients with no HCC diagnosis in the first 6 months post-SVR. This choice was justified by the need of excluding undiagnosed or occult HCC at the time of the antiviral treatment and clearly evaluate longterm HCC risk.

Altogether, more than 48,000 patients were included, 
Table 1 HCC risk after DAA treatment according to cirrhosis status, baseline FIB-4 and FIB-4 changes at 1-year

\begin{tabular}{lccc}
\hline Liver disease & $\begin{array}{c}\text { HCC risk with the baseline } \\
\text { FIB-4 (HCC \% per year) }\end{array}$ & FIB-4 at 1 y post-SVR & HCC risk (HCC \% per year) \\
\hline Cirrhosis & 1.16 & $<3.25$ & 2.3 \\
FIB-4 $<3.25$ & 3.66 & $<3.25$ & 2.45 \\
FIB-4 $\geq 3.25$ & & $\geq 3.25$ & 5.08 \\
No cirrhosis & & & $<3.25$ \\
FIB-4 $<3.25$ & 0.24 & $\geq 3.25$ & 0.24 \\
FIB-4 $\geq 3.25$ & 1.22 & $<3.25$ & 0.47 \\
\hline
\end{tabular}

DAA, direct antiviral agent; HCC, hepatocellular carcinoma; SVR, sustained viral response. Data from loannou et al. (13).

approximately $20 \%$ of them had liver cirrhosis and $60 \%$ were treated by DAA. Iannou et al. showed that baseline FIB-4 $\geq 3.25$ is an independent risk factor for HCC (Table 1). In patients with pre-treatment cirrhosis, HCC risk is more than 2-fold higher when baseline FIB-4 is $\geq 3.25$. In patients without cirrhosis, HCC risk is higher than $1 \% /$ year in patients with baseline FIB-4 $\geq 3.25$ with an adjusted risk 3 to 5 times higher compared to non-cirrhotic patients with baseline FIB-4 $<3.25$. During 10 -year follow-up, the HCC risk in IFN-treated cirrhotic patients did not significantly decrease over time in patients with baseline FIB-4 $\geq 3.25$ while remained lower than $1 \%$ in patients with baseline FIB-4 $<3.25$. The same findings, showing stability of HCC risk over-time in patients with FIB- $4 \geq 3.25$ and FIB-4 $<3.25$, were observed in the non-cirrhotic group. Data from the DAA-treated patients showed a reduction of HCC risk over time after SVR but the short follow-up (only 4 years) cannot allow to draw any firm conclusion. Changes in HCC risk following FIB-4 drop or increase after SVR were also calculated (Table 1). Cirrhotic patients with baseline high FIB-4 which dropped below 3.25 following SVR halved the HCC risk but this was still higher than 1.9\%/year. Cirrhotic patients with FIB-4 $<3.25$ which became $\geq 3.25$ after SVR, increased their risk of $2-3$ times which ranges to $1.35 \%$ to $2.75 \% / y e a r$. Non-cirrhotic patients with baseline FIB-4 $\geq 3.25$ who dropped to a FIB-4 $<3.25$ after SVR also halved their risk to $\sim 1 \% /$ year. Non-cirrhotic patients with baseline FIB-4 $<3.25$ who increased to a FIB- $4 \geq 3.25$ after treatment increased their HCC risk from 2 to 5 times but the HCC incidence was still lower to $1 \% /$ year.

The current international recommendations for HCC surveillance are based on cost-effectiveness analysis considering the costs per quality-adjusted life year (QALY) gained with the intervention. When the traditional costeffectiveness benchmark of $\$ 50,000 / \mathrm{QALY}$ is used, HCC surveillance in $\mathrm{HCV}$ patients is cost-effective in population with an HCC risk $\geq 1.5 \%$ /year. Using exclusively the presence of cirrhosis as discriminating factor, HCC surveillance in HCV could be strongly recommended only in cirrhotic patients. The data from Ioannou et al. suggest, however, that using FIB-4, it may be possible to stratify cirrhotic and non-cirrhotic patients into different groups of HCC risk to personalize post-SVR follow-up (Figure 1). Surveillance could be recommended for cirrhotic patients with FIB-4 $\geq 3.25$ (any time before and after SVR) and non-cirrhotic patients with FIB-4 $\geq 3.25$ that do not drop to $<3.25$ at 1 -year post-SVR. Non-cirrhotic patients with baseline FIB- $4<3.25$ and cirrhotic patients with FIB-4 constantly $<3.25$ could avoid surveillance. With the actual cost-effectiveness benchmark, this approach would change the follow-up of approximately $27 \%$ of cirrhotic patients (no surveillance recommended) and 4\% of non-cirrhotic patients (surveillance recommended) in the cohort of Ioannou and colleagues.

FIB- $4 \geq 3.25$ could help in refining the actual dichotomous assessment of HCC risk based on the diagnosis of liver cirrhosis because it has high specificity for advanced liver fibrosis (Metavir F3-F4) and it is higher in patients 
$\mathrm{HCC}$ risk after HCV treatment

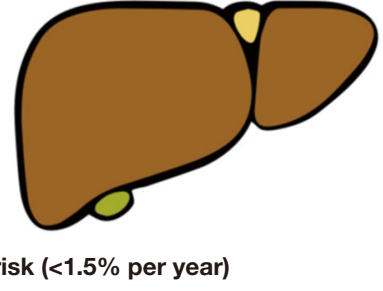

No cirrhosis and FIB4 $<3.25$ at any time

Cirrhosis with pre- and post-SVR FIB- $4<3.25$

No cost-effective surveillance

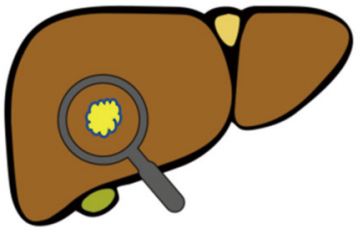

High risk $(\geq 1.5 \%$ per year)

Cirrhosis and FIB-4 $\geq 3.25$ at any time

No cirrhosis with pre- and post-SVR FIB-4 $\geq 3.25$

Cost-effective surveillance

Figure $1 \mathrm{HCC}$ risk after DAA treatment according to cirrhosis status and FIB-4. Cost-effectiveness is based on the traditional threshold of \$50,000/quality-adjusted life year gained. HCC, hepatocellular carcinoma; HCV, hepatitis C virus; DAA, direct antiviral agent; SVR, sustained viral response. Data from Ioannou et al. (13).

who are older, with portal hypertension and have higher transaminases. After SVR, the latter could be an indirect indicator of other concomitant liver diseases (e.g., NASH or alcohol abuse). Assessing the data by type of treatment, it appeared that HCC risk in DAA-treated patients with liver cirrhosis seemed to be higher than in IFN-treated cirrhotic patient. This could partly be explained by the inclusion of more advanced patients in the DAA group but a direct comparison of the HCC risk in the two groups was not the primary endpoint of the study and was not performed.

The need for HCC surveillance in F3 patients is uncertain and there are no strong recommendations for these patients $(5,6)$. Ioannou et al. showed that noncirrhotic patients with pre- and post-treatment FIB-4 $\geq 3.25$ have high risk of HCC and may benefit from surveillance. Considering that the category FIB- $4 \geq 3.25$ includes F3-F4 patients, these high-risk non-cirrhotic patients might have F3 fibrosis, indirectly suggesting that $\mathrm{F} 3$ patients can benefit from HCC surveillance. However, it is impossible to confirm this hypothesis and draw any conclusions on F3 patients since in this study FIB-4 was the only tool used to stratify patients for fibrosis level.

This study has some limitations that should be taken into account. Definition of cirrhosis is based on the ICD diagnosis record and not on pre-specified clinical criteria. Then, it is possible that some patients in the non-cirrhotic FIB-4 high group had unrecognized cirrhosis and conversely some patients in cirrhotic low FIB-4 group did not have cirrhosis. Moreover, no data on transient elastography or other noninvasive fibrosis biomarkers were available to confirm ICD diagnosis of cirrhosis, possibly further stratify patients and/ or verify the hypothesis that HCV F3 patients should be included in surveillance programs. Lastly, the follow-up of DAA treated patients is still short and confirmation from other cohorts, ideally with prospective data, are needed before change recommendations for HCC surveillance.

The data from Ioannou et al. reveal the complexity of the HCC risk in HCV patients after SVR and support the need for a better stratification that should not be based only on the presence of liver cirrhosis. The combination of cirrhosis, pre-treatment and post-SVR FIB-4 appears a promising strategy and more data are needed to confirm this approach.

\section{Acknowledgments}

Funding: This work was supported by Inserm, the University of Strasbourg, the European Union (ERC2014-AdG-671231-HEPCIR, 755460 -PRELICAN and 862551-HEPCAN) and Horizon 2020 research and innovation program under grant agreement 667273-HEPCAR, the Agence Nationale de Recherches sur le Sida et les Hépatites Virales (ANRS 15/1099), the French Cancer Agency (ARC IHU201301187 and IHU201901299), the US National Institutes of Health (NIH/NIAID U19AI123862-01, NIH/NIAID R03AI131066, NIH/NCI R21CA209940), the ITMO Cancer from AVIESAN in collaboration with INCa and Inserm (Plan Cancer "Single cell" 2018 grant) and the Office of the Assistant Secretary of Defense for Health Affairs through the PRCRP under 
Award No. W81XWH-16-1-0363. This work has been published under the framework of the LABEX [ANR-10LABX-0028_HEPSYS] and benefits from a funding from the state managed by the French National Research Agency as part of the Investments for the Future (Investissements d'Avenir) program. The content is solely the responsibility of the authors and does not necessarily represent the official views of the National Institutes of Health or the Department of Defense.

\section{Footnote}

Provenance and Peer Review: This article was commissioned by the editorial office, Hepatobiliary Surgery and Nutrition. The article did not undergo external peer review.

Conflicts of Interest: Conflicts of Interest: All authors have completed the ICMJE uniform disclosure form (available at http://dx.doi.org/10.21037/hbsn.2020.01.05). The authors have no conflicts of interest to declare.

Ethical Statement: The authors are accountable for all aspects of the work in ensuring that questions related to the accuracy or integrity of any part of the work are appropriately investigated and resolved.

Open Access Statement: This is an Open Access article distributed in accordance with the Creative Commons Attribution-NonCommercial-NoDerivs 4.0 International License (CC BY-NC-ND 4.0), which permits the noncommercial replication and distribution of the article with the strict proviso that no changes or edits are made and the original work is properly cited (including links to both the formal publication through the relevant DOI and the license). See: https://creativecommons.org/licenses/by-nc-nd/4.0/.

\section{References}

1. El-Serag HB. Epidemiology of viral hepatitis and hepatocellular carcinoma. Gastroenterology 2012;142:1264-73.e1.

2. Carrat F, Fontaine H, Dorival C, et al. Clinical outcomes in patients with chronic hepatitis $\mathrm{C}$ after direct-acting antiviral treatment: a prospective cohort study. Lancet 2019;393:1453-64.

3. Kanwal F, Kramer J, Asch SM, et al. Risk of Hepatocellular Cancer in HCV Patients Treated With Direct-Acting Antiviral Agents. Gastroenterology 2017;153:996-1005.e1.

4. Hamdane N, Juhling F, Crouchet E, et al. HCV- induced Epigenetic Changes Associated With Liver Cancer Risk Persist After Sustained Virologic Response. Gastroenterology 2019;156:2313-29.e7.

5. EASL Clinical Practice Guidelines: Management of hepatocellular carcinoma. J Hepatol 2018;69:182-236.

6. Heimbach JK, Kulik LM, Finn RS, et al. AASLD guidelines for the treatment of hepatocellular carcinoma. Hepatology 2018;67:358-80.

7. Sterling RK, Lissen E, Clumeck N, et al. Development of a simple noninvasive index to predict significant fibrosis in patients with HIV/HCV coinfection. Hepatology 2006;43:1317-25.

8. Kanwal F, Kramer J, Asch SM, et al. Risk of Hepatocellular Cancer in HCV Patients Treated With Direct-Acting Antiviral Agents. Gastroenterology 2017;153:996-1005.e1.

9. Suh B, Park S, Shin DW, et al. High liver fibrosis index FIB-4 is highly predictive of hepatocellular carcinoma in chronic hepatitis B carriers. Hepatology 2015;61:1261-8.

10. Tobari M, Hashimoto E, Taniai M, et al. The characteristics and risk factors of hepatocellular carcinoma in nonalcoholic fatty liver disease without cirrhosis. J Gastroenterol Hepatol 2020;35:862-9.

11. Kim JH, Lee M, Park SW, et al. Validation of modified fibrosis-4 index for predicting hepatocellular carcinoma in patients with compensated alcoholic liver cirrhosis. Medicine (Baltimore) 2018;97:e13438.

12. Moon AM, Green PK, Berry K, et al. Transformation of hepatitis $\mathrm{C}$ antiviral treatment in a national healthcare system following the introduction of direct antiviral agents. Aliment Pharmacol Ther 2017;45:1201-12.

13. Ioannou GN, Beste LA, Green PK, et al. Increased Risk for Hepatocellular Carcinoma Persists Up to 10 Years After HCV Eradication in Patients With Baseline Cirrhosis or High FIB-4 Scores. Gastroenterology 2019;157:1264-78.e4.

14. Reig M, Mariño Z, Perelló C, et al. Unexpected high rate of early tumor recurrence in patients with $\mathrm{HCV}$-related HCC undergoing interferon-free therapy. J Hepatol 2016;65:719-26.

15. Conti F, Buonfiglioli F, Scuteri A, et al. Early occurrence and recurrence of hepatocellular carcinoma in HCVrelated cirrhosis treated with direct-acting antivirals. J Hepatol 2016;65:727-33.

Cite this article as: Saviano A, Tripon S, Baumert TF. FIB-4 score and hepatocellular carcinoma risk after hepatitis $\mathrm{C}$ virus cure: time to revise surveillance? HepatoBiliary Surg Nutr 2020;9(5):661-664. doi: 10.21037/hbsn.2020.01.05 